\title{
Prevalence of complicated carotid atherosclerotic plaques ispilateral to ischemic cryptogenic stroke using high-resolution mri
}

Tobias Saam*, Tobias Freilinger ${ }^{1}$, Andreas Schindler ${ }^{1}$, Jochen Grimm¹, Caroline Schmidt ${ }^{1}$, Fabian Bamberg ${ }^{1}$, Martin Dichgans ${ }^{1}$, Chun Yuan ${ }^{2}$, Maximilian F Reiser ${ }^{1}$, Konstantin Nikolaou ${ }^{1}$

From 2011 SCMR/Euro CMR Joint Scientific Sessions

Nice, France. 3-6 February 2011

\section{Introduction}

Although distinct pathogenetic mechanisms for ischemic stroke have long been recognized, a definite or even probable etiology can not be established in about one third of all patients ("cryptogenic strokes"). Recent studies have shown that high-resolution carotid MRI is able to identify complicated American Heart Association lesion type VI (AHA-LT6) with hemorrhage, thrombus or rupture of the fibrous cap with good correlation to histopathology.

\section{Purpose}

The purpose of our study was to evaluate the prevalence of AHA-LT6 in carotid arteries of subjects with cryptogenic stroke.

\section{Methods}

30 consecutive patients ( 24 men, mean age $69.9 \pm$ 11.9 years) with cryptogenic stroke and intimal thickening by duplex sonography were recruited from our stroke unit. All patients underwent extensive clinical

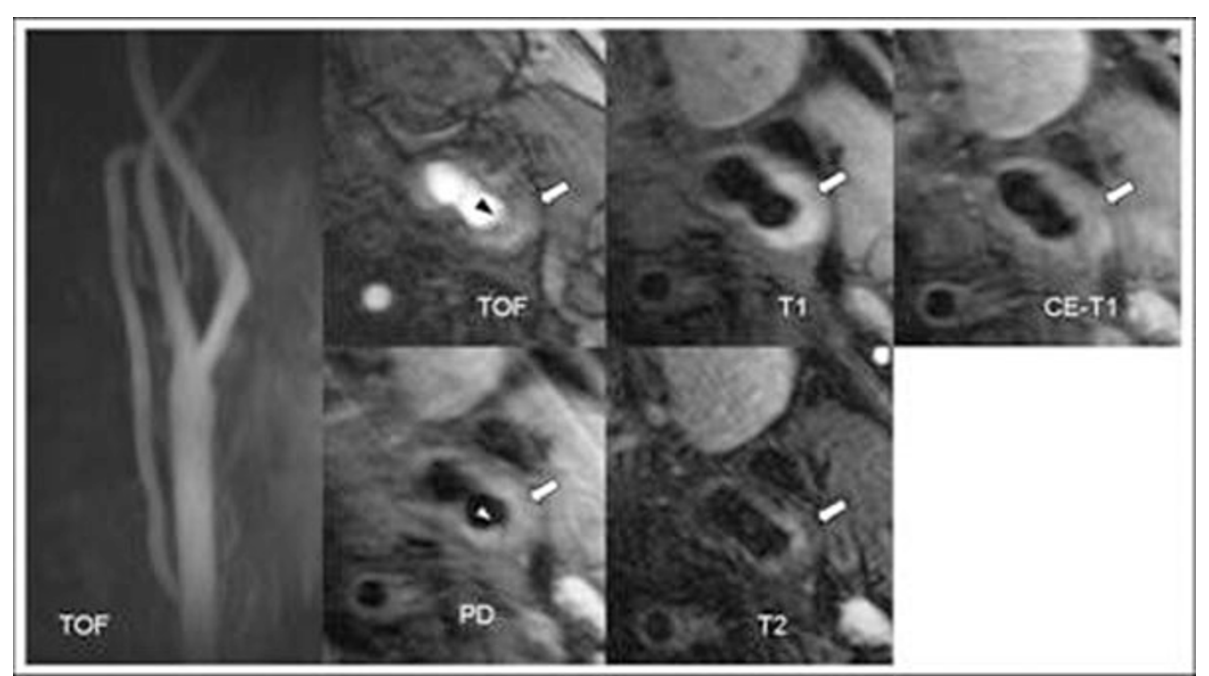

Figure 1 Images of a non-obstructive complicated AHA lesion type VI of a 66-year old male patient with a "cryptogenic"stroke ipsilateral to the lesion. The triangles on the TOF and PDW Images point to the site of the plaque rupture. The arrows point to a large lipid/necrotic core with type I Hemorrhage which does not cause substantial luminal narrowing (see TOF images on the left).

'University of Munich, Munich, Germany

Full list of author information is available at the end of the article

(c) 2011 Saam et al; licensee BioMed Central Ltd. This is an open access article distributed under the terms of the Creative Commons 
workup (lab, brain MRI, duplex sonography, 24-hour ECG, transesophageal echocardiography) to exclude carotid stenosis $\geq 50 \%$, cardiac embolism, small vessel disease and other causes of stroke. All subjects received a high-resolution carotid black-blood MRI at 3.0-Tesla with fat-saturated pre- and post-contrast T1w-, PDw-, T2w- and TOF images using surface coils and Parallel Imaging techniques (PAT factor $=2$ ). Prevalence of AHA-LT6 was determined in both carotid arteries based on previously published MRI criteria by two experienced reviewers who were blinded to the clinical information.

\section{Results}

AHA-LT6 with hemorrhage, rupture of the fibrous cap and / or thrombus were found in 14 out of 30 arteries (46.7\%) ipsilateral and in 1 out of 30 arteries (3.3\%) contralateral to the ischemic, "cryptogenic" stroke $(P<0.001)$. Of the 15 plaques classified as AHA-LT6, 13 had plaque hemorrhage, 2 had mural thrombi and 8 a rupture of the fibrous cap. Figure 1.

\section{Conclusions}

Complicated non-stenotic carotid atherosclerotic lesions were found significantly more often ipsilateral than contralateral to ischemic "cryptogenic" stroke.

\footnotetext{
Author details

${ }^{1}$ University of Munich, Munich, Germany. ${ }^{2}$ University of Washington, Seattle, WA, USA.
}

Published: 2 February 2011 